Review

\title{
Therapeutic Apheresis in Prevention and Treatment of Antibody- Mediated Rejection of Renal Allografts
}

Maurizio Salvadori ${ }^{1,+, *}$, Aris Tsalouchos ${ }^{2,+}$

1. Department of Renal Transplantation, Careggi University Hospital, viale Pieraccini 18, 50139 Florence, Italy; E-Mail: maurizio.salvadori1@gmail.com

2. Aris Tsalouchos Nephrology and Dialysis Unit, Saints Cosmas and Damian Hospital, Via Cesare Battisti, 2-51017 Pescia (PT), Italy; E-Mail: aris.tsalouchos@gmail.com

+ These authors contributed equally to this work.

* Correspondence: Maurizio Salvadori; E-Mail: maurizio.salvadori1@gmail.com

Academic Editor: Haval Shirwan

Special Issue: Antibody Mediated Rejection in Organ Transplantation

\section{OBM Transplantation}

2019 , volume 3 , issue 4

doi:10.21926/obm.transplant.1904093
Received: October 13, 2019

Accepted: November 25, 2019

Published: December 02, 2019

\begin{abstract}
Antibody-mediated rejection represents a significant barrier to favorable long-term outcomes after kidney transplantation and remains the most common cause of allograft failure. Therapeutic apheresis techniques are commonly used, in combination with other treatments such as immunosuppressive drugs, in the pre-transplant and post-transplant protocols for the prevention and treatment of antibody-mediated injury. The rationale is to remove the donor-specific antibodies and the other inflammatory mediators, which include cytokines, chemokines, and complement degradation molecules, and it is also related to immunomodulatory effects which determine an increased susceptibility of cell-mediated and humoral immunity to immunosuppressive agents. In the present review article, current knowledge regarding the use of therapeutic apheresis in combination with the known and emerging biological immunosuppressive therapies, for the prevention and management of antibody-mediated rejection, has been discussed.
\end{abstract}

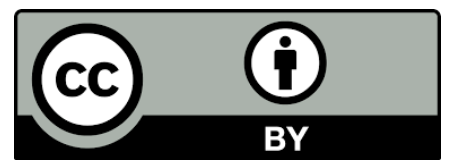

(C) 2019 by the author. This is an open access article distributed under the conditions of the Creative Commons by Attribution License, which permits unrestricted use, distribution, and reproduction in any medium or format, provided the original work is correctly cited. 


\section{Keywords}

Antibody-mediated rejection; kidney transplantation; therapeutic apheresis

\section{Introduction}

Antibody-mediated rejection (AMR) is known to be a severe complication arising after kidney transplantation (KT), with potentially deleterious effects on graft survival. Currently, AMR is widely recognized as a continuous process, with varying degrees of activity and damage, clinically as well as histologically, expressed with multiple phenotypes which are now identified and termed as acute AMR, subclinical AMR, and chronic AMR [1, 2].

The presence of preformed anti-HLA antibodies in the transplant candidate represents a major immunological barrier to a successful KT. Despite the prevention of this condition with desensitization protocols, up to one-third of the highly sensitized recipients may develop AMR following the transplantation [3, 4]. Therefore, the ability to successfully deliver incompatible transplants and optimize the long-term outcomes is dependent on the ability to successfully approach and manage an AMR. AMR presents a significant burden in the case of non-sensitized individuals as well, as de novo early or late DSA (dnDSA) may emerge following the KT [5].

Increasing evidence suggests that the prevention and treatment of AMR requires a combination of strategies rather than a single approach. In this context, therapeutic apheresis (TA) techniques are principally employed as an adjunctive therapeutic tool to the immunosuppressive agents in protocols, for preventive preoperative procedures as well as during the post-transplant period in case of development of AMR (Table 1). TA techniques that are used widely include therapeutic plasma exchange (TPE) and selective TA techniques such as double-filtration plasmapheresis (DFPP), immunoadsorption (IA), and extracorporeal photopheresis (ECP) [6].

Table 1 Therapeutic apheresis in the prevention and treatment of antibody-mediated rejection of the renal allograft.

Key points:

- Therapeutic apheresis techniques:

- Therapeutic plasma exchange,

- Double-filtration plasmapheresis,

- Immunoadsorption,

- Extracorporeal photopheresis.

- Apheresis is always performed in conjunction with other immunosuppressive drugs (IVIG, rituximab, \pm additional immunosuppression)

- Indications:

- Desensitization of deceased-donor kidney transplant recipients: low-quality evidence available that are based on observational studies or case series

- Desensitization of living-donor kidney transplant recipients: moderate-quality evidence based on RCTs with important limitations or exceptionally strong evidence from observational studies

- Treatment of antibody-mediated rejection: moderate-quality evidence based on RCTs with important limitations or exceptionally strong evidence from observational studies 
In the present review, the rationale and the evidence supporting the application of TA for the prevention and treatment of AMR of renal allograft has been examined, and the currently used therapeutic protocols have been discussed.

\section{Therapeutic Apheresis in the Prevention of Antibody-Mediated Rejection}

Approximately $30 \%$ of the KT candidates exhibit detectable anti-HLA antibodies, and approximately half of them exhibit high sensitization with HLA antibody reactivity to over $80 \%$ of the potential donors (Panel Reactivity Antibody $\geq 80 \%$ ) [7].

Sensitization occurs when the transplant candidate develops immunological memory to donor's antigens from prior transplants, blood transfusions, and pregnancies [8, 9].

KT with the presence of donor-specific anti-HLA antibodies (DSAs) at pre-transplant is referred to as HLA-incompatible transplantation. After transplantation, the presence of DSAs in high amounts, typically in the form of antibodies against donor HLAs, detected with positive complement-dependent cytotoxicity (CDC) crossmatch, results in hyperacute rejection. Whereas, the presence of DSA in small amounts, detected using Luminex solid-phase assay with mean fluorescence intensity $(\mathrm{MFI})>3000$, leads to reduced graft survival by causing acute AMR and/or chronic humoral rejection [10,11]. Therefore, candidates exhibiting high sensitization encounter difficulty in finding a crossmatch-negative donor kidney, ending up exhausted due to waiting on the list for an acceptable match. According to Fuggle et al. [12], sensitized candidates remain on the waiting list for a compatible donor kidney two to three times longer than the non-sensitized KT candidates. The possibilities for a highly sensitized candidate who is waiting on the deceaseddonor transplant list are higher after undergoing a desensitization protocol and further better in the cases where a living donor is already available. In this context, TA has a central role to play as an anti-humoral therapeutic strategy.

\subsection{Desensitization of Deceased-Donor Kidney Transplant Recipients}

The currently available desensitization protocols commonly use a combination of high-dose intravenous immunoglobulin (IVIG) and Rituximab (RTX) in order to lower the titers of the preformed HLA-antibodies in the candidates on the waiting list, which increases the probability of finding an acceptable deceased-donor [13]. However, in a recent, randomized, placebo-controlled, double-blind clinical trial, IVIG combined with RTX did not exhibit efficiency in reducing the MFI of the immunodominant anti-HLA donor-specific antibody in the patients with chronic AMR [14].

TA (TPE or IA), if performed while on the waiting list, has historically been demonstrated to reduce long waiting times in case of highly sensitized candidates [15-17]. Such strategies, however, are not always effective and may present risks associated with extended immunosuppression on the dialysis.

The data available regarding the efficacy of reduction in the amount of preformed anti-HLA antibodies in preventing hyperacute rejection, acute $A M R$, and late transplant glomerulopathy through peri-pre-transplant TPE in the deceased-donor KT (DDKT) are limited [18-20]. Beimler et al. [18] were pioneer in reporting a successful DDKT in two crossmatch-positive recipients using a single peri-pre-transplant TPE session and RTX. Cold ischemic time (CIT) in the therapeutic protocol was not prolonged as TPE during the transport of the kidneys from the donor center to the transplant center. Post desensitization, the crossmatch turned negative, and to avoid an early 
rebound of DSAs, the TPE sessions during the post-transplant period were extended until stable allograft function was achieved [18]. Both the patients exhibited satisfactory graft outcomes two years after the KT [18]. Using the same desensitization protocol, the same research group reported excellent short-term and medium-term outcomes in a larger cohort of 12 DDKTs with positive cross-matches that turned negative after the desensitization [19]. Recently, a retrospective cohort study on DSA-positive recipients who had received DDKT demonstrated that a single peri-pre-transplant TPE session, in combination with anti-human thymocyte globulin (ATG) used for induction immunosuppression, did not result in a lower incidence of acute AMR within 6 months, in comparison to the DSA-positive recipients who had not received a TPE session [20]. Post-transplant TPE was not performed because the protocol included 3 to 5 days of ATG induction [20].

Loupy et al. [21], from the Paris group, reported the results of a combined post-transplant prophylactic IVIg/RTX/TPE treatment in DDKT with preformed DSAs and a negative crossmatch on the day of the transplant. These patients received 9 TPE sessions every alternate day after the transplantation, in addition to $2 \mathrm{~g} / \mathrm{kg}$ IVIg at Day 0, 2, 42, and 63 and RTX on Day 2 and 22. At 1year post-transplant, comparable results for patient and graft survival rates as well as the rate of acute AMR were obtained between the patients who received only IVIg and those who received IVIg, RTX, and TPE. However, the results for estimated glomerular filtration rate (e-GFR) were significantly worse, and proteinuria levels, as well as the rate of chronic AMR were observed to be significantly higher in the IVIg group [21]. These differences in the long-term functions were characterized by a significant decrease in the MFI of DSAs in the group of patients who received the more intensive post-transplant prophylactic regimen compared to those in the IVIg group [21]. Recently, the Paris group reported the long-term outcomes of a high immunological risk program which included patients with high levels of peak DSA (MFI > 3000) and a negative crossmatch on the day of transplantation who received a post-transplant desensitization protocol with high-dose IVIg, TPE, and RTX. The results obtained for these patients were compared to those obtained for a control group which comprised patients who exhibited a lower immunological risk (MFI ranging between 500 and 3000) on the day of transplantation and who received post-transplant desensitization based on IVIg alone [22]. Patient survival was identical between these two groups. However, there were a significantly greater number of cases of acute T-cell rejection and AMR in the group with MFI > 3000, which clinically translates to significantly lower graft survival [22].

Semi-specific IA, which is aimed at preventing humoral graft injury, has also been employed, and mixed results have been reported. The Vienna transplantation center reported a favorable allograft outcome in a number of highly sensitized kidney transplant recipients after a peri-pretransplant IA session with a staphylococcal protein A column supplemented by repeated posttransplant treatment [23]. Subsequently, the same group reported that a single peri-pretransplant IA, in addition to pre-emptive ATG, could turn a positive crossmatch into a negative crossmatch, enabling a successful DDKT supported by a favorable long-term graft survival of 3 years [24]. The authors confirmed these data by extending their initial experience in a research paper published later [25]. Repeated post-transplant IA sessions were performed in this protocol to prevent a potentially harmful rebound of DSAs [24, 25]. In line with the Vienna group, Higgins et al. [26] reported a cohort with successful crossmatch conversion and prevention of hyperacute rejection through the use of peri-pre-transplant IA treatment. However, in this case, a considerably high graft loss rate was observed during the follow-up, with only $54 \%$ of the 
transplants surviving after a median follow-up of 26 months [26]. The difference in the outcome between these studies could be attributed to the significant differences between the desensitization protocols used in the two studies. Unlike the Vienna group, Higgins et al. did not repeat the post-transplant IA sessions [24-26]. In addition, the Vienna group [24, 25], in order to prevent an exaggerated increase of $\mathrm{CIT}$, excluded transplantation for the patients for whom a negative crossmatch could not be obtained through treatment with $6 \mathrm{~L}$ of plasma, while Higgins et al. [26] prescribed greater than $30 \mathrm{~L}$ of plasma volume to convert a positive crossmatch in a few patients, which resulted in significant increases in CIT (up to $62 \mathrm{~h}$ ). Recently, the Vienna group reported that one-third of the 101 DSA-positive recipients of DDKT underwent intense IA-based desensitization and experienced acute $A M R$, and that the DSA MFI levels were significantly associated with acute rejection (20\% versus $71 \%$ AMR rates at $<5000$ versus $>15,000$ peak DSA MFI) [27]. The 3-year graft survival rate in the DSA-positive recipients was reported to be significantly lower than that in the DSA-negative recipients (79\% vs. $88 \%$; $P=0.008$ ) [27].

These data highlight the significant prognostic value of MFI levels and suggest that the intensification of the TA treatment in post-transplant desensitization protocols must be customized according to MFI levels.

Currently, the eighth special issue of the American Society for Apheresis (ASFA) guidelines, according to the above-stated observational studies and case series, provides a weak recommendation (Grade $2 \mathrm{C}$ ) and a weak category description (III: Optimum role of apheresis therapy has not been established; decision making should be individualized) for the use of TA techniques in the desensitization of DDKT recipients [6].

\subsection{Desensitization of Living-Donor Kidney Transplant Recipients}

In the case of sensitized candidates who have incompatible living donor available, paired donor exchange (PDE) is the best alternative to select. However, for most of the highly sensitized candidates, the probability of finding a match in the relatively small pool of donors available in the PDE programs is low, and desensitization alone or in combination with PDE represents almost the only viable option for transplantation [28]. HLA-incompatible desensitized living-donor KT (LDKT) exhibits significantly lower graft survival in comparison to HLA-compatible LDKT [29]. The results of multicenter studies indicate, however, that it is worth desensitizing the HLA-incompatible patients who have a living potential donor, because such patients have significantly better longterm survival after KT compared to the highly sensitized candidates on a KT waiting list who did not receive a kidney from a deceased donor [30-32].

TA holds a central position in the currently available desensitization protocols and is also strongly recommended by the current ASFA guidelines, although with moderate-quality evidence (Grade 1B) [6].

The most commonly used protocol is an alternate-day TPE followed by the use of low-dose IVIg (100-150 mg/kg) prior to transplantation [30-34]. Most transplant centers also commence antirejection medications, tacrolimus, and mycophenolate mofetil (MMF), up to 2 weeks prior to the surgery [35]. Montgomery et al. [30] demonstrated, in the largest series of HLA desensitization based on TPE plus low-dose IVIg, that at 5-year follow-up, a significantly greater survival was achieved in patients who received LDKT (90.6\%) compared to those who remained on dialysis (51.5\%) or those who were placed on a DDKT waiting list with or without KT (65.6\%). On average, 
patients received $4 \pm 4$ TPE treatments prior to LDKT and $5 \pm 4$ TPE treatments after the LDKT [30]. More recently, in a larger multicenter $(n=22)$ study conducted in US involving 1,025 patients, Orandi et al. [31] validated the results reported by the Baltimore research group [30].

Magee et al. [36] also reported their experience with TPE/low-dose IVIG plus RTX in 28 crossmatch-positive patients. The AMR rate was observed to be high (39\%), although the mean serum creatinine levels $(1.5 \mathrm{mg} / \mathrm{dL})$ remained satisfactory within a mean follow-up of 22 months and only three grafts were lost. Similar results for the application of TPE/low-dose IVIg plus RTX in 51 transplanted patients have been reported by the University of Illinois [37]. The acute rejection rate in these patients was determined to be $33 \%$, with optimal graft survival at 2 years (93\%).

Morath et al. [38] examined the effect of adding one dose of RTX $\left(375 \mathrm{mg} / \mathrm{m}^{2}\right)$ just prior to the KT together with IA performed prior to and after the transplantation. After a median of 10 IA treatments, all ten patients were reported to be desensitized successfully and transplanted. The recipients also received a median of seven post-transplant IA treatments. After a median followup of 19 months, the reversible AMR rate was observed to be $30 \%$, and the patient and allograft survival rates were $100 \%$ and $90 \%$, respectively, with a mean serum creatinine level of $1.6 \mathrm{mg} / \mathrm{dL}$ [38]. Similar results were observed with the use of RTX plus IA as reported recently by Kauke et al. [39] who conducted a study on a small series of 8 LDKT recipients. Klein et al. [40], in a study conducted on a series of 23 sensitized patients, performed pre-transplant IA sessions together with the use of tacrolimus, MMF, and steroids, with the goal of achieving an MFI $<1000$ on the day of transplantation. On Day 0 and 1, the recipients also received one dose of RTX. The induction therapy was based on either ATG or basiliximab, and the IA sessions were continued posttransplantation until the serum creatinine level of $<2 \mathrm{mg} / \mathrm{dL}$ was achieved and MFI stabilized at the value of $<1000$. This desensitization protocol resulted in excellent outcomes at 2-year followup, with a graft survival rate of $100 \%$ and a median serum creatinine level of $1.42 \mathrm{mg} / \mathrm{dL}$ [40]. In order to allow LDKT in six highly sensitized patients, Rostaing et al. [41] performed an IA-based desensitization protocol plus IVIg, RTX, and ATG as induction therapy. This protocol effectively reduced or eliminated the DSAs in $71 \%$ of the recipients at the time of transplant. Three recipients manifested AMR, although the long-term renal function was satisfactory.

Woodle et al. [42] applied an alternative protocol that incorporated TPE, the proteasome inhibitor bortezomib, and RTX, resulting in a significant decrease in the DSAs in both LDKT and DDKT, achieving successful transplantation in 19 among the 44 highly sensitized patients and low acute rejection rates (18.8\%) at the time point of 6 months.

In a recent review, Malvezzi et al. [43] proposed an algorithm based on pre-transplant MFI levels for the use of various TA techniques in the desensitization protocols. The authors suggested that the use of TPE should be restricted to cases where the highest pre-transplant MFI is $\leq 9000$. In such circumstances, TPE should be delivered on a daily basis until the MFI is $\leq 3000$. MFI must be assessed after every five sessions. If the MFI of the DSA is between 9000 and 13000, DFPP could be implemented on a daily basis. The rationale of this may be explained by the fact that, compared to TPE, DFPP allows larger volumes of plasma to be treated. When the target of MFI < 9000 is achieved, DFPP may be replaced by TPE. In the event of MFI > 12000 prior to the commencement of the desensitization, IA has to be applied on a daily basis, as DFPP proves to be insufficient in such cases. When the MFI is reduced $(<6000)$, IA could be replaced by DFPP or TPE to obtain an MFI threshold of $\sim 3000$. The usage of DFPP instead of going directly for IA when MFI is >9000 could be attributed to the higher cost of the IA technique. However, in certain 
circumstances, the rate of DFPP sessions could be limited by the fibrinogen level, for example, when the fibrinogen level prior to a DFPP session is $<1 \mathrm{~g} / \mathrm{L}$. The authors concluded that in all these scenarios, as soon as the MFI is reduced to a value <3000, KT may be performed, as the DSA strength would be low in that case.

It is suggested that on the basis of the current studies and regardless of the cost of each TA technique, the best strategy would be to apply IA plus RTX until the MFI becomes $<3000$. The addition of IVIG might also be relevant in this setting.

\section{Therapeutic Apheresis in Treatment of Antibody-Mediated Rejection}

Early acute AMR may be severe and may result in graft loss, although it is also potentially responsive to the currently available treatments [2]. On the contrary, late acute AMR (occurring at greater than 6 months post-transplant) may present a mixed cellular and humoral rejection and is often non-responsive to the current treatment methods, such as in case of chronic AMR, and in certain cases, subclinical AMR. Late acute and chronic AMR may result from dnDSA formation, the incomplete elimination of DSA following an earlier acute AMR episode, or the persistence of preformed DSA even after the desensitization [2].

TA, as an adjunctive therapeutic option, holds a central position in the treatment of AMR. In addition to the removal of DSAs, TA techniques remove cytokines, adhesion molecules, and circulating complement regulatory components [44]. Moreover, evidence suggests that TA has immunomodulatory effects as well. TA has been associated with a variety of autoimmune diseases involving a decline in B cells and natural killer (NK) cells, and an increase in T cells, T suppressor cell function, and regulatory $T$ cells (Tregs) [45-48]. The immunomodulatory effects of TA determine the increased susceptibility of cell-mediated and humoral immunity to the immunosuppressive agents, and numerous therapeutic protocols integrate the administration of these agents with TPE during the treatment of AMR in order to enhance their immunosuppressive effects.

The ASFA guidelines strongly recommend the use of TA for the treatment of AMR, although the quality of evidence provided by the current studies is moderate (Grade 1B) [6].

\subsection{Therapeutic Apheresis and Intravenous Immunoglobulin}

When acute AMR occurs, TPE or IA plus IVIG and increased immunosuppression are considered the standard of care (SOC) treatment currently, as it may be used to decrease the antibody levels and arrest the rejection process in majority of the patients [2].

In a recent meta-analysis by Wan et al. [49], it was demonstrated, on the basis of 5 RCTs, that graft survival after antibody removal with TPE or IA resulted in no benefit in the trials with a shorter follow-up (1-7 months) [50,51], while the trials with a longer follow-up (2-5 years) exhibited a trend toward a benefit [52-54].

In a recent retrospective cohort study investigating the use of TPE plus IVIG in the treatment of late AMR, with approximately $50 \%$ of the patients having chronic histology lesions, Lee et al. [55] demonstrated an improvement in graft survival in the intervention group compared to the control group which did not receive any therapy, in a mean follow-up of 7 years. In contrast, Einecke et al. [56] observed no effect on graft survival after treatment with TPE plus IVIG in late AMR, with approximately $63 \%$ of the patients having chronic histology lesions. 
In conclusion, according to the currently available data, the basis of establishing TPE plus IVIG as SOC treatment in AMR lacks strong evidence, and a high-quality RCT with sufficient power to evaluate the efficacy of this treatment would provide the much-required reassurance on this delicate topic. However, it is improbable that such a trial would be conducted due to the ethical perplexity of enrolling patients in a no-treatment group, which has been historically associated with high risks of graft failure.

\subsection{Add-On Treatments to Therapeutic Apheresis and Intravenous Immunoglobulin}

Different add-on treatments in the current SOC treatment have been proposed over time by different transplant centers according to their preference $[2,49]$.

The use of RTX in acute AMR presented promising results as reported by several small-scale retrospective series $[57,58]$. In the first non-randomized study using RTX plus TPE/IVIG vs. IVIG alone, Lefaucheur et al. [59] concluded that high-dose IVIG is inferior to combination therapy. However, it was practically not possible to determine which among RTX or TPE led to the improvement [59].

In addition, two retrospective cohort studies compared RTX plus TPE/IVIG to TPE/IVIG or IVIG alone, and both reported an improvement in graft survival in the RTX group [60,61]. The patients in the RTX group, however, received a higher dose of TPE and IVIG, limiting the ability to provide a direct comparison between the groups.

In a small multicenter double-blind RCT, comparing RTX plus TPE/IVIG to placebo plus TPE/IVIG for the treatment of acute AMR, Sautenet et al. [62] demonstrated that no additional benefit was achieved with the use of RTX in graft survival after 1 year. However, the 1-year follow-up period may not have been sufficiently long to identify a difference in graft survival. Recently, Oblak et al. [63], within the limitations associated with a retrospective cohort study, confirmed that there was no evidence of any benefit of the addition of RTX to the SOC treatment for AMR in a longer followup period (2 years).

Bortezomib, a proteasome inhibitor, in several non-randomized retrospective studies and case reports, has been described to benefit the treatment of acute AMR when used in combination with TPE and IVIG $[64,65]$ or with TPE and RTX [66], while certain studies have reported no improvement in e-GFR with the use of bortezomib as an add-on therapy with TPE and IVIG for late AMR [67].

The single RCT comparing the use of bortezomib in combination with TPE and ATG vs. TPE, RTX, and ATG or TPE and ATG alone, in patients with mixed AMR and acute cellular rejection, reported observing no difference in graft survival among the three groups [68].

The complement inhibitor eculizumab, a humanized monoclonal $\lg G$ antibody that binds to the complement protein $\mathrm{C} 5$ and inhibits the formation of $\mathrm{MAC}$, and $\mathrm{C} 1-\mathrm{INH}$, a serine protease inhibitor that inactivates both $\mathrm{C} 1 \mathrm{r}$ and $\mathrm{C} 1 \mathrm{~s}$, thereby inhibiting the first step of the complement cascade, have also been evaluated in combination with TPE and IVIG for the treatment of AMR.

Locke et al. [69] were pioneers in reporting the use of eculizumab in combination with TPE and IVIG to treat severe AMR, demonstrating a reversal of the AMR episode. In a study conducted with 24 patients who developed severe oliguric AMR after HLA-incompatible LDKT, Orandi et al. [70] demonstrated that a combination of splenectomy and eculizumab and RTX as an add-on therapy 
to TPE/IVIG resulted in effective intervention for rescuing and preserving allograft function, compared to the use of splenectomy alone or eculizumab alone as the add-on therapy.

In an RCT in which 18 patients with acute AMR were subjected to C1-INH (Cinryze) plus TPE/IVIG or placebo plus TPE/IVIG, Montgomery et al. [71] reported reduced transplant glomerulopathy at 6 months in the C1-INH group. A multicenter phase III RCT (NCT02547220) evaluating C1-INH as an add-on therapy to TPE/IVIG or IA/IVIG has concluded recently and the results are awaited to be published.

In conclusion, various add-on treatment options are being employed with current SOC treatment according to their targets in the steps of AMR pathogenesis, with different results. Future RCTs should assess definitive endpoints, and until that happens, the regimen to be followed should be considered on a case-to-case basis.

\subsection{Extracorporeal Photopheresis}

Extracorporeal Photopheresis (ECP) is a cell therapy procedure that begins with the separation of peripheral white blood cells (WBCs) and non-nucleated cells from the plasma using centrifugation. Subsequently, the isolated suspension of WBCs is subjected to extracorporeal treatment using 8-methoxypsoralen (8-MOP), followed by exposure to ultraviolet A (UVA) light prior to reinfusion in the patient [72]. The combination of 8-MOP and UV-A results in the crosslinking of the pyrimidine bases in DNA, leading to the apoptosis of lymphoid cells, largely T-cells and the natural killer (NK) cells [73]. Upon reinfusion, apoptotic lymphoid cells are phagocytosized by immature dendritic antigen-presenting cells (iDCs), which subsequently undergo maturation and present the self-antigens in a pro-tolerant signaling environment [74]. The activated T cells differentiate into several cell lineages, particularly Tregs, which mediate a specific immunological tolerance through the induction of anergy or apoptosis in the self-reactive lymphocytes [74].

Over the years, indications for ECP have increased as it promotes anti-inflammatory and tolerogenic responses without causing global immunosuppression [75]. In solid organ transplantation, ECP has been successfully applied to treat acute heart allograft rejection and chronic allograft dysfunction after lung transplantation [76, 77]. In addition, ECP has been used as a part of calcineurin inhibitor (CNI) sparing protocols to reduce the drug side effects such as nephrotoxicity and the neurological or infectious complications [78].

A limited number of reports are available on the use of ECP in chronic AMR. Sunder-Plassman et al. [79], who employed intensive long-term ECP treatments (2 consecutive procedures every 2 weeks for 17 cycles), reported benefit in treating a single patient with chronic rejection. Dall'Amico et al. [80] reported progressive improvement in renal function and consecutive biopsy specimens during the course of application of ECP for treating one patient with chronic rejection. In contrast, Horina et al. [81] reported no response in treating two patients with an approach involving two consecutive ECP procedures per month for three months.

Finally, ECP has also been employed as a preventive treatment, as reported by a small case series, with favorable outcomes, i.e., rejection did not occur in any of the treated patients and there was a notable increase in circulating Tregs [82].

The current ASFA guidelines [6] do not consider this issue, as the relevant data are old and no RCT is available. Further investigation and evidence on the usefulness of ECP in the prevention and treatment of $A M R$ are required. 


\section{Conclusions and Future Direction}

TA, with the variety of its techniques, represents a cornerstone in the prevention and treatment of AMR. However, the preferred protocol has not been established so far, neither for the sensitized candidates nor the transplant recipients with acute AMR, which remains a major cause of KT failure. Currently, the majority of the transplant centers use protocols based on their own experience, often with results conflicting with each other due to the retrospective design of most of these studies and the limited number of participants in them. In the era of emerging novel biological immunosuppressive therapies with increasing number of specific actions and immune targets directed against cell-surface antigens or plasma-soluble molecules, the concerns such as the optimal timing and dosage in the apheresis sessions and the preferred adjunctive immunosuppressive therapy to TA remain to be challenging for the transplantation community. Future therapeutic protocols, the ones that best address these open issues, for achieving better clinical outcomes in the prevention and treatment of AMR, should emerge from well-designed multicenter clinical trials.

\section{Author Contributions}

Maurizio Salvadori and Aris Tsalouchos contributed equally to this work.

\section{Funding}

No fund has been received for this manuscript.

\section{Competing Interests}

The authors have declared that no competing interests exist.

\section{References}

1. Loupy A, Haas M, Solez K, Racusen L, Glotz D, Seron D, et al. The Banff 2015 Kidney Meeting Report: Current challenges in rejection classificationand prospects for adopting molecular pathology. Am J Transplant. 2017; 17: 28-41.

2. Montgomery RA, Loupy A, Segev DL. Antibody-mediated rejection: New approaches in prevention and management. Am J Transplant. 2018; 18: 3-17.

3. Loupy A, Suberbielle-Boissel C, Hill GS, Lefaucheur C, Anglicheau D, Zuber J, et al. Outcome of subclinical antibody-mediated rejection in kidney transplant recipients with preformed donor-specific antibodies. Am J Transplant. 2009; 9: 2561-2570.

4. Archdeacon P, Chan M, Neuland C, Velidedeoglu E, Meyer J, Tracy L, et al. Summary of FDA antibody-mediated rejection workshop. Am J Transplant. 2011; 11: 896-906.

5. Velidedeoglu E, Cavaillé-Coll MW, Bala S, Belen OA, Wang Y, Albrecht R. Summary of 2017 FDA Public Workshop: Antibody-mediated rejection in kidney transplantation. Transplantation. 2018; 102: e257-e264.

6. Padmanabhan A, Connelly-Smith L, Aqui N, Balogun RA, Klingel R, Meyer E, et al. Guidelines on the use of therapeutic apheresis in clinical practice - evidence-based approach from the 
Writing Committee of the American Society for Apheresis: The eighth special issue. J Clin Apher. 2019; 34: 171-354.

7. Sethi S, Choi J, Toyoda M, Vo A, Peng A, Jordan SC. Desensitization: Overcoming the immunologic barriers to transplantation. J Immunol Res. 2017; 2017: 6804678.

8. Obrador GT, Macdougall IC. Effect of red cell transfusions on future kidney transplantation. Clin J Am Soc Nephrol. 2013; 8: 852-860.

9. Sanfilippo F, Vaughn WK, Bollinger RR, Spees EK. Comparative effects of pregnancy, transfusion, and prior graft rejection on sensitization and renal transplant results. Transplantation. 1982; 34: 360-366.

10. McKenna RM, Takemoto SK, Terasaki PI. Anti-HLA antibodies after solid organ transplantation. Transplantation. 2000; 69: 319-326.

11. Mohan S, Palanisamy A, Tsapepas D, Tanriover B, Crew RJ, Dube G, et al. Donor-specific antibodies adversely affect kidney allograft outcomes. J Am Soc Nephrol. 2012; 23: 2061-2071.

12. Fuggle SV, Martin S. Tools for human leukocyte antigen antibody detection and their application to transplanting sensitized patients. Transplantation. 2008; 86: 384-390.

13. Vo AA, Choi J, Cisneros K, Reinsmoen N, Haas M, Ge S, et al. Benefits of rituximab combined with intravenous immunoglobulin for desensitization in kidney transplant recipients. Transplantation. 2014; 98: 312-319.

14. Moreso F, Crespo M, Ruiz JC, Torres A, Gutierrez-Dalmau A, Osuna A, et al. Treatment of chronic antibody mediated rejection with intravenous immunoglobulins and rituximab: $A$ multicenter, prospective, randomized, double-blind clinical trial. Am J Transplant. 2018; 18: 927-935.

15. Palmer A, Taube D, Welsh K, Bewick M, Gjorstrup P, Thick M. Removal of anti-HLA antibodies by extracorporeal immunoadsorption to enable renal transplant. Lancet. 1989; 1: 10-12.

16. Hiesse C, Kriaa F, Rousseau P, Farahmand H, Bismuth A, Fries D, et al. Immunoadsorption of antiHLA antibodies for highly sensitized patients awaiting renal transplantation. Nephrol Dial Transplant. 1992; 7: 944-951.

17. Alarabi A, Backman U, Wikström B, Sjöberg O, Tufveson G. Plasmapheresis in HLAimmunosensitized patients prior to kidney transplantation. Int J Artif Organs. 1997; 20: 51-56.

18. Beimler JH, Morath C, Schmidt J, Ovens J, Opelz G, Rahmel A, et al. Successful deceaseddonor kidney transplantation in crossmatch-positive patients with peritransplant plasma exchange and Rituximab. Transplantation. 2009; 87: 668-671.

19. Morath C, Beimler J, Opelz G, Ovens J, Scherer S, Schmidt J, et al. An integrative approach for the transplantation of high-risk sensitized patients. Transplantation. 2010; 90: 645-653.

20. Tipjaiaue $P$, Ingsathit $A$, Kantachuvesiri $P$, Rattanasiri $S$, Thammanichanond $D$, Mongkolsuk $T$, et al. Outcome of pretransplantation therapeutic plasma exchange in highly sensitized deceased-donor kidney transplant recipients. Transplant Proc. 2017; 49: 1249-1255.

21. Loupy A, Suberbielle-Boissel C, Zuber J, Anglicheau D, Timsit MO, Martinez F, et al. Combined posttransplant prophylactic IVIg/anti-CD 20/plasmapheresis in kidney recipients with preformed donor-specific antibodies: A pilot study. Transplantation. 2010; 89: 1403-1410.

22. Amrouche L, Aubert $O$, Suberbielle $C$, Rabant M, Van Huyen JD, Martinez F, et al. Long-term outcomes of kidney transplantation in patients with high levels of preformed DSA: The necker high-risk transplant program. Transplantation. 2017; 101: 2440-2448. 
23. Haas M, Böhmig GA, Leko-Mohr Z, Exner $M$, Regele $H$, Derfler $K$, et al. Peri-operative immunoadsorption in sensitized renal transplant recipients. Nephrol Dial Transplant. 2002; 17: 1503-1508.

24. Lorenz M, Regele H, Schillinger M, Kletzmayr J, Haidbauer B, Derfler K, et al. Peritransplant immunoadsorption: A strategy enabling transplantation in highy sensitized crossmatchpositive cadaveric kidney allograft recipients. Transplantation. 2005; 79: 696-701.

25. Bartel G, Wahrmann M, Regele H, Kikić Z, Fischer G, Druml W, et al. Peritransplant immunoadsorption for positive crossmatch deceased donor kidney transplantation. Am J Transplant. 2010; 10: 2033-2042.

26. Higgins RM, Bevan DJ, Carey BS, Lea CK, Fallon M, Bühler R, et al. Prevention of hyperacute rejection by removal of antibodies to HLA immediately before renal transplantation. Lancet. 1996; 348: 1208-1211.

27. Schwaiger E, Eskandary F, Kozakowski N, Bond G, Kikić Ž, Yoo D, et al. Deceased donor kidney transplantation across donor-specific antibody barriers: Predictors of antibody-mediated rejection. Nephrol Dial Transplant. 2016; 31: 1342-1351.

28. Montgomery RA, Lonze BE, Jackson AM. Using donor exchange paradigms with desensitization to enhance transplant rates among highly sensitized patients. Curr Opin Organ Transplant. 2011; 16: 439-443.

29. Bentall A, Cornell LD, Gloor JM, Park WD, Gandhi MJ, Winters JL, et al. Five-year outcomes in living donor kidney transplants with a positive crossmatch. Am J Transplant. 2013; 13: 76-85.

30. Montgomery RA, Lonze BE, King KE, Kraus ES, Kucirka LM, Locke JE, et al. Desensitization in HLA-incompatible kidney recipients and survival. N Engl J Med. 2011; 365: 318-326.

31. Orandi BJ, Luo X, Massie AB, Garonzik-Wang JM, Lonze BE, Ahmed R, et al. Survival benefit with kidney transplants from HLA-incompatible live donors. N Engl J Med. 2016; 374: 940-950.

32. Rostaing LP, Malvezzi P. HLA-incompatible kidney transplantation--Worth the risk? N Engl J Med. 2016; 374: 982-984.

33. Montgomery RA, Zachary AA. Transplanting patients with a positive donor-specific crossmatch: A single center's perspective. Pediatr Transplant. 2004; 8: 535-542.

34. Schweitzer EJ, Wilson JS, Fernandez-Vina M, Fox M, Gutierrez M, Wiland A, et al. A high panel-reactive antibody rescue protocol for cross-match-positive live donor kidney transplants. Transplantation. 2000; 70: 1531-1536.

35. Kumar V. Current status on the evaluation and management of the highly sensitized kidney transplant recipient. Curr Opin Nephrol Hypertens. 2015; 24: 570-575.

36. Magee CC, Felgueiras J, Tinckam K, Malek S, Mah H, Tullius S. Renal transplantation in patients with positive lymphocytotoxicity crossmatches: One center's experience. Transplantation. 2008; 86: 96-103.

37. Thielke JJ, West-Thielke PM, Herren HL, Bareato U, Ommert T, Vidanovic V, et al. Living donor kidney transplantation across positive crossmatch: The University of Illinois at Chicago experience. Transplantation. 2009; 87: 268-273.

38. Morath C, Beimler J, Opelz G, Scherer S, Schmidt J, Macher-Goeppinger S, et al. Living donor kidney transplantation in crossmatch-positive patients enabled by peritransplant immunoadsorption and anti-CD20 therapy. Transpl Int. 2012; 25: 506-517. 
39. Kauke T, Klimaschewski S, Schoenermarck U, Fischereder M, Dick A, Guba M, et al. Outcome after desensitization in HLA or ABO-incompatible kidney transplant recipients: A single center experience. PLoS One. 2016; 11: e0146075.

40. Klein K, Süsal C, Schäfer SM, Becker LE, Beimler J, Schwenger V, et al. Living donor kidney transplantation in patients with donor-specific HLA antibodies enabled by anti-CD20 therapy and peritransplant apheresis. Atheroscler Suppl. 2013; 14: 199-202.

41. Rostaing L, Congy N, Aarnink A, Maggioni S, Allal A, Sallusto F, et al. Efficacy of immunoadsorption to reduce donor-specific alloantibodies in kidney-transplant candidates. Exp Clin Transplant. 2015; 13: 201-206.

42. Woodle ES, Shields AR, Ejaz NS, Sadaka B, Girnita A, Walsh RC, et al. Prospective iterative trial of proteasome inhibitor-based desensitization. Am J Transplant. 2015; 15: 101-118.

43. Malvezzi $P$, Jouve $T$, Noble J, Rostaing L. Desensitization in the setting of HLA-incompatible kidney transplant. Exp Clin Transplant. 2018; 16: 367-375.

44. Reeves HM, Winters JL. The mechanisms of action of plasma exchange. Br J Haematol. 2014; 164: 342-351.

45. Yoshii F, Shinohara Y. Lymphocyte subset proportions in Guillain-Barrè syndrome patients treated with plasmapheresis. Eur Neurol. 2000; 44: 162-167.

46. Dau PC, Callahan JP. Immune modulation during treatment of systemic sclerosis with plasmapheresis and immunosuppressive drugs. Clin Immunol Immunopathol. 1994; 70: 159165.

47. De Luca G, Lugaresi A, larlori C, Marzoli F, Di lorio A, Gambi D, et al. Prednisone and plasma exchange improve suppressor cell function in chronic inflammatory demyelinating polyneuropathy. J Neruoimmunol. 1999; 95: 190-194.

48. Baráth $S$, Soltész $P$, Kiss E, Aleksza $M$, Zeher $M$, Szegedi $G$, et al. The severity of systemic lupus erythematosus negatively correlates with the increasing number of CD4+CD25(high)FoxP3+ regulatory $\mathrm{T}$ cells during repeated plasmapheresis treatments of patients. Autoimmunity. 2007; 40: 521-528.

49. Wan SS, Ying TD, Wyburn K, Roberts DM, Wyld M, Chadban SJ. The treatment of antibodymediated rejection in kidney transplantation: An updated systematic review and metaanalysis. Transplantation. 2018; 102: 557-568.

50. Allen NH, Dyer $P$, Geoghegan $T$, Harris $K$, Lee HA, Slapak M. Plasma exchange in acute renal allograft rejection. Transplantation. 1983; 35: 425-428.

51. Kirubakaran MG, Disney APS, Norman J, Pugsley DJ, Mathew TH. A controlled trial of plasmapheresis in the treatment of renal allograft rejection. Transplantation. 1981; 32: 164165.

52. Böhmig GA, Wahrmann $M$, Regele $H$, Exner $M$, Robl B, Derfler $K$, et al. Immunoadsorption in severe C4d-positive acute kidney allograft rejection: A randomized controlled trial. Am J Transplant. 2007; 7: 117-121.

53. Bonomini V, Vangelista A, Frascà GM, Di Felice A, D'Arcangelo GL. Effects of plasmapheresis in renal transplant rejection: A controlled study. Trans Am Soc Artif Intern Organs. 1985; 31: 698-703.

54. Blake $P$, Sutton $D$, Cardella CJ. Plasma exchange in acute renal transplant rejection. Prog Clin Biol Res. 1990; 337: 249-252. 
55. Lee CY, Lin WC, Wu MS, Yang CY, Yeh CC, Tsai MK. Repeated cycles of high-dose intravenous immunoglobulin and plasmapheresis for treatment of late antibody-mediated rejection of renal transplants. J Formos Med Assoc. 2016; 115: 845-852.

56. Einecke G, Bräsen J, Schwarz A, Haller H, Njau F. Treatment of late antibody-mediated rejection: Observations from clinical practice. Am J Transplant. 2016; 16: 609.

57. Faguer S, Kamar N, Guilbeaud-Frugier C, Fort M, Modesto A, Mari A, et al. Rituximab therapy for acute humoral rejection after kidney transplantation. Transplantation. 2007; 83: 12771280.

58. An GH, Yun J, Hong YA, Khvan M, Chung BH, Choi BS, et al. The effect of combination therapy with rituximab and intravenous immunoglobulin on the progression of chronic antibody mediated rejection in renal transplant recipients. J Immunol Res. 2014; 2014 : 828732.

59. Lefaucheur C, Nochy D, Andrade J, Verine J, Gautreau C, Charron D, et al. Comparison of combination plasmapheresis/IVIg/anti-CD20 versus high dose IVIg in the treatment of antibody-mediated rejection. Am J Transplant. 2009; 9: 1099-1107.

60. Kaposztas Z, Podder H, Mauiyyedi S, Illoh O, Kerman R, Reyes M, et al. Impact of rituximab therapy for treatment of acute humoral rejection. Clin Transplant. 2009; 23: 63-73.

61. Lefaucheur C, Loupy A, Vernerey D, Duong-Van-Huyen JP, Suberbielle C, Anglicheau D, et al. Antibody-mediated vascular rejection of kidney allografts: A population-based study. Lancet. 2013; 381: 313-319.

62. Sautenet B, Blancho G, Büchler M, Morelon E, Toupance O, Barrou B, et al. One-year results of the effects of rituximab on acute antibody-mediated rejection in renal transplantation: RITUX ERAH, a multicenter double-blind randomized placebo-controlled trial. Transplantation. 2016; 100: 391-399.

63. Oblak T, Lindič J, Gubenšek J, Kveder R, Aleš Rigler A, Škoberne A, et al. Treatment of antibody-mediated rejection of kidney grafts with bortezomib and/or rituximab compared to standard regimen: Experience of Slovene National Center. Clin Nephrol. 2017; 88: 91-96.

64. Waiser J, Budde K, Schütz M, Liefeldt L, Rudolph B, Schönemann C, et al. Comparison between bortezomib and rituximab in the treatment of antibody-mediated renal allograft rejection. Nephrol Dial Transplant. 2012; 3: 1246-1251.

65. Flechner SM, Fatica R, Askar M, Stephany BR, Poggio E, Koo A, et al. The role of proteasome inhibition with bortezomib in the treatment of antibody-mediated rejection after kidney-only or kidney-combined organ transplantation. Transplantation. 2010; 12: 1486-1492.

66. Walsh RC, Everly JJ, Brailey P, Rike AH, Arend LJ, Mogilishetty G, et al. Proteasome inhibitorbased primary therapy for antibody-mediated renal allograft rejection. Transplantation. 2010; 3: 277-284.

67. Gupta G, Abu Jawdeh BG, Racusen LC, Bhasin B, Arend L, Trollinger B, et al. Late antibodymediated rejection in renal allografts: Outcome after conventional and novel therapies. Transplantation. 2014; 97: 1240-1246.

68. Govil A, Mogilishetty G, Cardi M, Kremer J, Cuffy M, Paterno F, et al. Randomized controlled trial of mixed acute rejection therapy in renal allografts: 3 year follow up. Am J Transplant. 2016; 16: 601.

69. Locke JE, Magro CM, Singer AL, Segev DL, Haas M, Hillel AT, et al. The use of antibody to complement protein $\mathrm{C} 5$ for salvage treatment of severe antibody-mediated rejection. Am J Transplant. 2009; 9: 231-235. 
70. Orandi BJ, Zachary AA, Dagher NN, Bagnasco SM, Garonzik-Wang JM, Van Arendonk KJ, et al. Eculizumab and splenectomy as salvage therapy for severe antibody-mediated rejection after HLA-incompatible kidney transplantation. Transplantation. 2014; 98: 857-863.

71. Montgomery RA, Orandi BJ, Racusen L, Jackson AM, Garonzik-Wang JM, Shah T, et al. Plasmaderived $\mathrm{C} 1$ esterase inhibitor for acute antibody-mediated rejection following kidney transplantation: Results of a randomized double-blind placebo-controlled pilot study. Am J Transplant. 2016; 16: 3468-3478.

72. Perotti C, Sniecinski I. A concise review on extracorporeal photochemotherapy: Where we began and where we are now and where are we going! Transfus Apher Sci. 2015; 52: 360-368.

73. Ratcliffe N, Dunbar NM, Adamski J, Couriel D, Edelson R, Kitko CL, et al. American Society for Apheresis. National Institutes of Health State of the Science Symposium in Therapeutic Apheresis: Scientific opportunities in extracorporeal photopheresis. Transfus Med Rev. 2015; 29: 62-70.

74. Cho A, Jantschitsch C, Knobler R. Extracorporeal photopheresis-an overview. Front Med (Lausanne). 2018; 5: 236.

75. Marques MB, Adamski J. Extracorporeal photopheresis: Technique, established and novel indications. J Clin Apher. 2014; 29: 228-234.

76. Barten MJ, Dieterlen MT. Extracorporeal photopheresis after heart transplantation. Immunotherapy. 2014; 6: 927-944.

77. Hachem R, Corris P. Extracorporeal photopheresis for bronchiolitis obliterans syndrome after lung transplantation. Transplantation. 2018; 102: 1059-1065.

78. Jaksch P, Knobler R. ECP and solid organ transplantation. Transfus Apher Sci. 2014; 50: 358362.

79. Sunder-Plassman G, Druml W, Steininger R, Hönigsmann H, Knobler R. Renal allograft rejection controlled by photopheresis. Lancet. 1995; 346: 506.

80. Dall'Amico R, Murer L, Montini G, Andreetta B, Zanon GF, Zacchello G, et al. Successful treatment of recurrentrejection in renal transplant patients with photopheresis. J Am Soc Nephrol. 1998; 9: 121-127.

81. Horina JH, Müllegger RR, Horn S, Holzer H, Halwachs G, Kerl H, et al. Photopheresis for renal allograft rejection. Lancet. 1995; 346: 61.

82. Lamioni A, Carsetti R, Legato A, Landolfo A, Isacchi G, Emma F, et al. Induction of regulatory $T$ cells after prophylactic treatment with photopheresis in renal transplant recipients. Transplantation. 2007; 83: 1393-1396. 


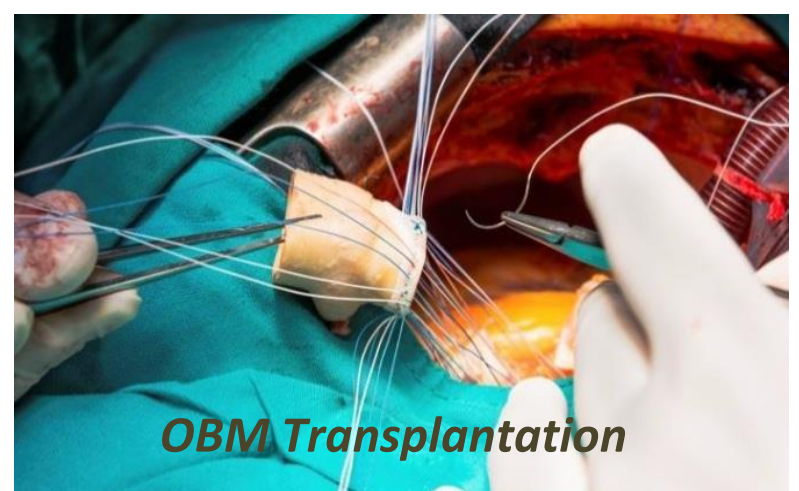

Enjoy OBM Transplantation by:

1. Submitting a manuscript

2. Joining in volunteer reviewer bank

3. Joining Editorial Board

4. Guest editing a special issue

For more details, please visit:

http://www.lidsen.com/journals/transplantation 